\title{
Associatively activated representations of food events resemble food outcome expectancies more closely than they resemble food-based memories
}

\author{
DANIEL C. LINWICK \\ University of Wisconsin, River Falls, Wisconsin \\ and \\ J. BRUCE OVERMIER \\ University of Minnesota, Minneapolis, Minnesota
}

\begin{abstract}
When Pavlovian stimuli activate representations of food, do these representations resemble memories of food consumed in the recent past or expectancies of food that is imminent? In Experiments $1 \mathrm{~A}$ and $1 \mathrm{~B}$, this question was addressed by training pigeons on a symbolic matching-to-sample task involving different grains as memory cues or as expectancy cues for correct choices. Autoshaping trials involving these same grains were interspersed among matching-to-sample trials, as were test trials involving the substitution of autoshaping stimuli for cues in the matching-to-sample task. Control over choices transferred to autoshaping stimuli in both experiments, suggesting that associatively activated representations of food resemble both memories and expectancies. In Experiment 2, pigeons were trained on a symbolic matching-to-sample task in which food and no-food memory cues (i.e., the samples) were juxtaposed with no-food and food expectancy cues. Subsequently, autoshaping stimuli, which activated representations of food and no food, were substituted for the samples. Choices by the pigeons indicated that associatively activated representations of food-related events resemble expectancies more closely than they do memories.
\end{abstract}

One procedure that is well suited to the study of representational processes is the symbolic delayed matching-tosample (MTS) task. In its simplest form with pigeons, this task involves two samples and two comparison stimuli. On trials initiated with one of the samples, choice responses to one comparison are correct, whereas on trials initiated with the other sample, choice responses to the other comparison are correct. The symbolic delayed MTS task involves a delay between the sample's removal and presentation of comparison stimuli, and choice accuracy declines precipitously with increasing delays (Grant, 1976). Notably, the delay-induced memory load in this task requires that a pigeon base its choices on a representational process of some kind.

A number of experiments have been conducted with the goal of identifying the nature of representations that mediate performance on the symbolic delayed MTS and

The work reported here was supported by the Center for Cognitive Sciences, University of Minnesota. Portions of Experiment 1A and 1B were presented at the Sixty-Seventh Annual Meeting of the Midwestern Psychological Association in Chicago, May 1995. Experiment 2 was presented at the Eleventh Annual Convention of the American Psychological Society in Denver, June 1999. We thank Gretchen M. Shay for her assistance with data collection. Correspondence concerning this article should be addressed to J. B. Overmier, Department of Psychology, University of Minnesota, N218 Elliott Hall, 75 East River Road, Minneapolis, MN 55455-0344 (e-mail: psyjbo@umn.edu). other delayed conditional choice tasks (for summaries of this research, see Grant, 1993; Honig \& Dodd, 1986; Riley, Cook, \& Lamb, 1981; Roitblat, 1984, 1993; Wasserman, 1986; Zentall, Hogan, \& Edwards, 1984; Zentall, Sherburne, \& Steirn, 1993; Zentall, Urcuioli, JacksonSmith, \& Steirn, 1991). One principle emerging from this research is well established: Animals are flexible in the coding strategies that they adopt in choice tasks requiring working memory (e.g., Cook, Brown, \& Riley, 1985; Urcuioli, Zentall, Jackson-Smith, \& Steirn, 1989; Zentall, 1998; Zentall, Steirn, \& Jackson-Smith, 1990; Zentall, Urcuioli, Jagielo, \& Jackson-Smith, 1989). Thus, when samples are easier to discriminate than comparison stimuli (e.g., when colors are used as samples and line orientation patterns as comparison stimuli), a pigeon may base its choices between comparison stimuli on a retrospective memory code - that is, on a representation of the sample. In contrast, when samples are harder to discriminate than comparison stimuli (e.g., when line orientation patterns are used as samples and colors as comparison stimuli), a pigeon may base its choices on a prospective memory code - that is, on a representation of the comparison stimulus to which it must respond (Roitblat, 1980; Zentall et al., 1991; Zentall et al., 1989). Importantly, for our purposes, when correct responses to different sample-comparison stimulus combinations are followed by unique outcomes, a pigeon may base its choices on another prospective memory codethat is, on a representation of these unique outcomes 
(Brodigan \& Peterson, 1976; Trapold, 1970; Trapold \& Overmier, 1972). Such representations are commonly referred to as differential outcome expectancies.

Although both rats and pigeons can flexibly use different coding strategies (Cook et al., 1985, and Zentall et al., 1990, respectively), an important and as yet unresolved issue surrounds the compatibility of retrospective and prospective processes in symbolic delayed MTS tasks. Are retrospective and prospective memory codes mutually exclusive, or might certain circumstances engender both types of coding? Given a symbolic delayed MTS task involving food and no food as samples and these same events as unique outcomes for correct responses to different comparison stimuli, for instance, might a pigeon encode both pre- and postchoice food-related events? If so, might the memory codes (mediational processes) corresponding to these events interact?

In a series of experiments designed to assess such possibilities, Peterson, Linwick, and Overmier (1987) trained four groups of pigeons on a symbolic delayed MTS task. Different colors and different food-based events served as elements of compound samples. Line patterns served as comparison stimuli. The correspondence between the color element of a sample and the correct comparison stimulus was the same for all four groups: On trials beginning with green, vertical was correct, and on trials beginning with red, horizontal was correct. However, two factors distinguished the groups: (1) the relationship between the food-based element of the sample (food or no food) and the correct comparison stimulus and (2) the relationship between the color element of the sample and the food-related outcome of a correct choice response (food or no food; see Table 1). It should be noted that the event we label as no food here for expository convenience was, in fact, a stimulus associated with food delivery (illumination of the grain aperture), but without the food.

Because the color element and the food-based element of the sample were correlated with the correct comparison stimulus in the memory (MEM) and the memory plus expectancy (MEM+EXP) groups, either (or both) of these elements could have cued choices. In the same way, both

Table 1

Relationship Between the Events Making Up a Symbolic Delayed MTS Trial and the Correct Comparison Stimulus in Peterson, Linwick, and Overmier (1987)

\begin{tabular}{lccc}
\hline Group & $\begin{array}{c}\text { Color } \\
\text { Element } \\
\text { of Sample }\end{array}$ & $\begin{array}{c}\text { Food-Based } \\
\text { Element } \\
\text { of Sample }\end{array}$ & $\begin{array}{c}\text { Food Outcome } \\
\text { Event }\end{array}$ \\
\hline COL & yes & no & no \\
MEM & yes & yes & no \\
EXP & yes & no & yes \\
MEM+EXP & yes & yes & yes \\
\hline
\end{tabular}

Note- "Yes" indicates a correlation between a particular event and the correct comparison stimulus and the potential for control over choice responses by this event. "No" indicates the absence of a correlation. COL, color memory only; MEM, color memory and food-based memory; EXP, color memory and food outcome expectancy; MEM+EXP, color memory, food-based memory, and food outcome expectancy. the color element of the sample and the food outcome event were correlated with the correct comparison stimulus in the EXP and MEM+EXP groups. Therefore, representations of either (or both) events - memories of the color sample or expectancies of food outcome events - could have cued choices in these groups. Importantly, the MEM+EXP condition was divided into two subgroups: one in which the pre- and the postchoice food-related events were congruent (i.e., both were food or both were no food on any given trial) and another in which they were always incongruent. The performance of the two subgroups in this condition was of particular interest, because the subgroups were designed to reveal the interaction of retrospective and prospective codes.

The performance of the four groups and the relative performance of the two subgroups forced several conclusions, among them the following: (1) Food outcome expectancies are more powerful than food-based memories as mediators of delayed conditional choice performance; (2) food-based memories and food outcome expectancies do interact under certain conditions; and (3) animals discriminate, at least to some degree, directly activated memories from associatively activated expectancies. Otherwise, the pigeons would not have been able to master the symbolic delayed MTS task when food-based memories and food outcome expectancies were incongruent.

Experiments 1 and 2 were performed to further assess how associatively activated representations of foodrelated events relate to memory and expectancy processes, using a transfer-of-control design.

\section{EXPERIMENT 1}

Transfer-of-control designs have proven useful in studying mediational processes (Overmier \& Lawry, 1979; Rescorla \& Solomon, 1967; Trapold \& Overmier, 1972). Urcuioli (1990, Experiment 3B), for example, used transfer of control to examine the contribution of differential outcomes to a pigeon's performance in a symbolic delayed MTS task. For one group in this experiment, correct choices on the different symbolic-matching trials were reinforced with unique outcomes (i.e., food and no food). Subsequent to mastering the matching task, the pigeons advanced to a phase in which two novel colors were paired with the same food and no-food outcomes as those used in the first phase. Finally, during the transfer phase, the novel colors were substituted for the vertical and the horizontal samples used in symbolic matching, and training resumed. The novel colors exerted immediate control over the correct choices. Urcuioli (1990) ascribed this transfer of control to differential outcome expectancies that were common between the symbolic-matching and the pairing phases of his study.

In a related study, Steirn, Jackson-Smith, and Zentall (1991, Experiment 2) used a differential autoshaping procedure to condition expectancies of food and no food to two different colors. The food and no-food events employed during differential autoshaping subsequently served as 
samples in a symbolic delayed MTS task. Finally, Steirn et al. assessed transfer in the third phase of their experiment by substituting the colors used in autoshaping (Phase 1) for the food and no-food samples used during symbolicmatching training. Although the level of transfer was modest, expectancies of food and no food elicited by the different colors appeared to mediate the evocation of responses to the correct comparison stimuli. Thus, associatively activated expectancies of food-related events evoked the same choice responses as those that directly activated food-based memories had evoked in original training.

Our present study assessed the capacity of associatively activated representations of food-related events to substitute for two kinds of cues: (1) memories of food-based samples and (2) expectancies of food outcomes. The experiments described below differed from the experiments of Urcuioli (1990) and Steirn et al. (1991) in several respects. Different grains (safflower and milo) were used to assess the mediational properties of internal representations. The use of two grains reduced the likelihood that different overt responses would develop to the stimuli with which they were correlated and thus ensured that memories and expectancies of food events, not differential responding, would serve as mediators of choices. In the pairing phase of Experiment 1, which was used to endow novel stimuli with mediational properties, autoshaping trials were interspersed among symbolic delayed MTS trials. Finally, the mediational properties of memories and expectancies of safflower and milo were assessed with unreinforced transfer-of-control probe trials. These probe trials involved the stimuli used in autoshaping and the comparison stimuli from the symbolic delayed MTS task. Would representations of food-related events activated associatively by autoshaping stimuli more closely resemble the directly activated memories (Experiment 1A) or the associatively activated expectancies (Experiment 1B)?

\section{Method}

\section{Subjects}

The subjects were 6 female White Carneaux retired breeder pigeons from Palmetto Pigeon Plant in Sumter, South Carolina. The subjects were individually caged in a colony with a 14:10-h light: dark cycle. Two weeks after their introduction to the colony, they were reduced to $80 \%$ of their ad lib weight. Water and grit were freely available throughout the experiment.

\footnotetext{
Apparatus

The apparatus consisted of three identical fan-ventilated operant chambers $(28 \times 23.5 \times 29 \mathrm{~cm}, 1 \times \mathrm{w} \times \mathrm{h})$. One-plane projectors were mounted behind each of three response keys, which were configured in a row across the front wall in each chamber. The projectors enabled the presentation of various stimuli, including geometric forms, red, green, blue, and yellow fields, and three parallel black lines on a white field with either a vertical or a horizontal orientation. The response keys $(2.5 \mathrm{~cm}$ in diameter, $8 \mathrm{~cm}$ center to center) were located approximately $20 \mathrm{~cm}$ above the floor. Six centimeters above the floor beneath the center response key was an aperture for accessing a food tray. A second aperture and food tray were located beneath the right response key. When one of these feeders operated, its tray was raised for $3 \mathrm{sec}$, making one of two different grains available, and the aperture was illuminated with white light. Notably, the particular feeder from which a particular grain was available varied
}

across sessions. Background noises were attenuated by enclosing each chamber in a cubicle. A masking noise of $80 \mathrm{~dB}$ came from a speaker within the cubicle. The chambers were lit with a 4-W ceiling lamp.

\section{Procedure}

Because the two experiments (1A and 1B) had substantial commonalities, all the procedures will be described together to the degree possible.

Preliminary training (autoshaping). The pigeons in Experiment 1A $(n=3)$ and Experiment 1B $(n=3)$ were trained to eat from the center and the side feeders over a series of magazine training sessions. They were then autoshaped to respond to a white field whenever this stimulus was projected onto one of the three response keys. Autoshaping trials were presented according to a variable time 60 -sec schedule. The subjects were presented 36 trials per session, and they received a maximum of 6 autoshaping sessions.

Symbolic delayed MTS training. Preliminary training was followed by symbolic delayed MTS training. Figure 1 presents the different arrangements of events that made up the conditional choice trials. In the memory condition in Experiment 1A, the beginning of an MTS trial was demarcated by flashing the houselight off and on at a rate of $2.5 \mathrm{cps}$ for $2 \mathrm{sec}$. This event was replaced by a compound sample stimulus that consisted of (1) a set of vertical lines projected onto the center response key, followed by the operation of the feeder containing safflower, or (2) a set of horizontal lines projected onto the center key, followed by the operation of the feeder containing milo. The durations of the line and food elements of the sample stimulus were 5 and $3 \mathrm{sec}$, respectively; the pigeons were not required to peck either element to progress through the trial.

On every MTS trial, the sample stimulus (vertical-safflower or horizontal-milo) was followed by the projection of red and green fields onto the left and right response keys. The specific side on which these comparison stimuli appeared was random. On the trials beginning with safflower, the color green was arbitrarily designated the correct stimulus, whereas on the trials beginning with milo, red was correct. A single peck to the key on which the correct color appeared extinguished both colors and resulted in 3 -sec access to food. Safflower and milo were equally likely following correct choices on both kinds of trial; that is, the two grains were presented randomly following correct choices on the 24 trials beginning with the vertical-safflower stimulus and on the 24 trials beginning with the horizontal-milo stimulus. The intertrial interval (ITI) was $15 \mathrm{sec}$. The subjects received 48 trials of symbolic delayed MTS training per session.

Whereas correct choices resulted in immediate access to food, incorrect choices initiated a correction sequence. Thus, a single peck to the incorrect color extinguished the two comparison stimuli and produced a 30-sec blackout. At the end of this blackout, the line component of the sample stimulus, but not the food component, was again presented. The latter was not included in the correction sequence, in order to ensure that incorrect choices would not serve to increase the total number of food presentations per session. The line component was followed by presentation of only the correct color, and a response to this stimulus resulted in access to safflower or milo.

The symbolic delayed MTS training procedure was similar for the subjects assigned to the expect condition in Experiment 1B. Thus, the houselight flashed off and on at the beginning of every trial and was replaced by a compound sample stimulus. The latter consisted of two elements: (1) the operation of a feeder containing grain, followed by a set of vertical lines on the center response key, or (2) the operation of a feeder, followed by a set of horizontal lines on the center key. Notably, safflower and milo preceded both the vertical and the horizontal lines with equal probability. Hence, in the expect condition, the line element of the sample, but not the food-based element of the sample, was related to the correct choice response.

The compound sample stimulus was followed by the projection of red and green fields onto the left and right response keys. On the trials involving vertical lines, the color green was arbitrarily desig- 
Phase 1: Events Making Up Symbolic Delayed MTS Trials During Initial Training

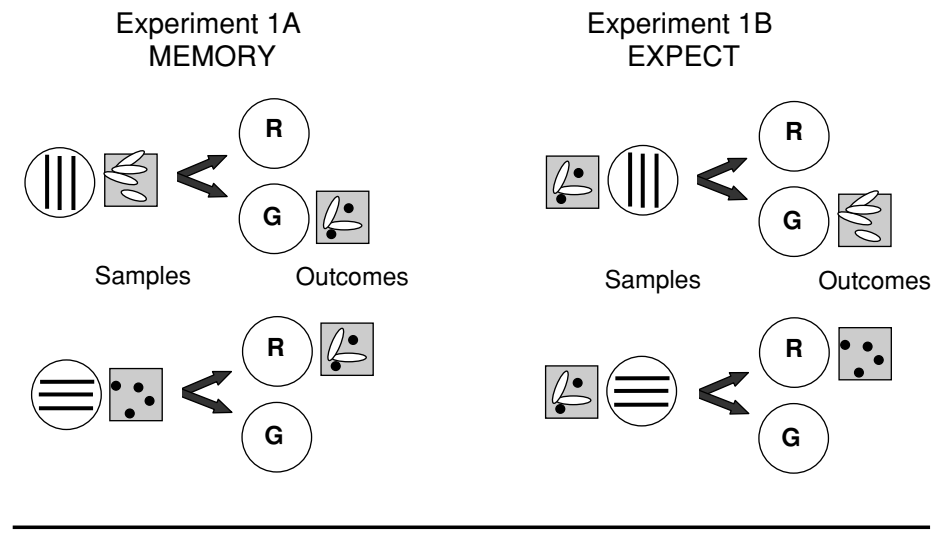

Phase 2 for Both Experiments 1A and 1B: Discriminative Autoshaping Trials

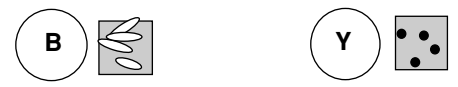

Phase 3 for Both Experiments 1A and 1B: Transfer Testing

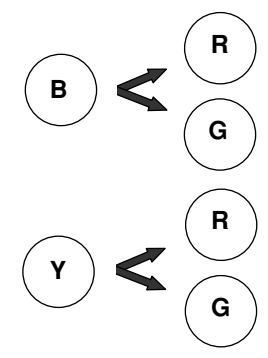

Figure 1. Experiment 1: Three phases of the transfer-of-control design. The light, oblong features depicted in squares are safflower; the dark, round features are milo. R, red; G, green; B, blue; Y, yellow.

nated the correct stimulus, whereas on the trials involving horizontal lines, red was correct. A single peck to the key on which the correct color appeared extinguished both colors and produced a food outcome. Correct choices on the 24 vertical trials were always reinforced with safflower, and correct choices on the 24 horizontal trials were always reinforced with milo. The two responses thus produced different food outcomes.

As per the memory condition in Experiment 1A, whereas correct choices resulted in immediate access to food, followed by an ITI, incorrect choices initiated a correction sequence. A single peck to the incorrect color thus extinguished the two comparison stimuli and produced a blackout. At the end of the blackout, the houselight was turned on, and the line component of the sample stimulus was again presented. This component was, in turn, followed by the presentation of only the correct color. A response to the latter produced the same outcome as that programmed for a correct choice - that is, safflower on vertical-green correction sequences and milo on horizontal-red correction sequences.

Symbolic delayed MTS training and discriminative autoshaping. Symbolic delayed MTS training continued until a subject's average choice accuracy exceeded $88 \%$ for two consecutive 6 -session blocks. Subsequent to meeting criterion, the subjects received concurrent discriminative autoshaping and symbolic delayed MTS training. Thus, 16 discriminative autoshaping trials were randomly interspersed among the 48 MTS trials, for a total of 64 trials per session $($ ITI $=15 \mathrm{sec})$. Autoshaping trials began with the illumination of the center response key with either a blue or a yellow field. The duration of the blue and yellow stimuli was $5 \mathrm{sec}$, and they were followed by 3 -sec access to safflower and milo, respectively. These two types of trials were equal in number.

The experimental protocol called for a progression from discriminative autoshaping concurrent with symbolic delayed MTS training with a delay of $0 \mathrm{sec}$ to discriminative autoshaping concurrent with symbolic delayed MTS training with a delay of $1 \mathrm{sec}$, then $2 \mathrm{sec}$, and then $4 \mathrm{sec}$, and finally with mixed delays. Indeed, no subject required more than 7 sessions to progress from 0 -sec delay to 1 -sec delay conditions. Because the performance of several pigeons deteriorated before the 4-sec delay was inserted, however, discriminative autoshaping was discontinued after a maximum of 32 sessions of MTS training with a delay of $1 \mathrm{sec}$ or greater. Thus, all the subjects were returned to 0 -sec delay conditions and retrained to a criterion of $90 \%$ correct for one 3 -session block. They then advanced to symbolic delayed MTS training with mixed delays.

Symbolic delayed MTS training with mixed delays and discriminative autoshaping. The conditions of symbolic delayed MTS training with a delay of $0 \mathrm{sec}$ and with mixed delays were identical, with one exception. During mixed-delay training, the delay between the removal of the sample and the presentation of the comparison 
stimuli varied unpredictably across trials. Delays of $0,1,2$, and $4 \mathrm{sec}$ were used, and there were 48 trials per session - 6 involving the vertical sample at each delay and 6 involving the horizontal sample. As a precaution against a deterioration in choice performance concomitant with the transition from MTS training with a delay of $0 \mathrm{sec}$ to MTS training with mixed delays, pairs of mixed-delay sessions alternated with pairs of 0 -sec delay refresher sessions. This regimen continued until the subjects had completed 6 sessions of mixeddelay training, whereupon discriminative autoshaping trials were reintroduced. Discriminative autoshaping was conducted concurrently with symbolic delayed MTS training in both the mixed-delay and the refresher sessions. Pairs of mixed-delay sessions continued to alternate with pairs of refresher sessions until the subjects had completed an additional 6 sessions of symbolic delayed MTS training with mixed delays.

Probe test. The probe test was designed to assess whether the discriminative autoshaping stimuli had acquired control over conditional choices by virtue of their association with grains that were common between the autoshaping and the symbolic delayed MTS tasks. Except for the introduction of a small number of probe trials, the conditions of training during this phase of the experiment were identical to those just described.

A probe trial consisted of the illumination of the center response key with a blue or a yellow field for $5 \mathrm{sec}$. One, 2 , or $4 \mathrm{sec}$ following the offset of this stimulus, a pigeon was offered a choice between red and green fields on the side keys. The side on which these colors appeared was random, and a response to one of them extinguished both colors and initiated the ITI. Extinction contingencies were in effect on these trials, as opposed to the symbolic delayed MTS and discriminative autoshaping trials. Therefore, performance on probe trials did not engender the learning of new sample-correct-comparison relations and afforded a conservative estimate of the strength of any transfer effect.

The first probe trial of a session was presented after a minimum of 6 symbolic delayed MTS and 2 discriminative autoshaping trials. There was a total of 70 trials per session: 48 symbolic delayed MTS, 16 discriminative autoshaping, and 6 probe trials (one per probe stimulus per delay). Pairs of sessions involving MTS training with mixed delays concurrent with discriminative autoshaping and probes alternated with pairs of refresher sessions; 6 probe test sessions were administered.

\section{Results and Discussion}

Our analyses focus on two related questions. First, did control over choices between the comparison stimuli transfer from the samples used in the symbolic delayed MTS task to the discriminative autoshaping stimuli? And second, was there a difference between food-based memories (Experiment 1A) and food outcome expectancies (Experiment 1B) in the degree of transfer supported?

\section{Acquisition}

The pigeons assigned to the memory condition and the expect condition required a mean of 60.7 sessions $(S E=$ 8.97 ) and 62.0 sessions $(S E=9.85)$, respectively, to meet the criterion for symbolic delayed MTS training with a delay of $0 \mathrm{sec}$. Thus, the subjects in Experiments $1 \mathrm{~A}$ and 1B mastered the conditional choice task at comparable rates.

\section{Baseline}

Figure 2 displays the mean percentage of correct symbolic delayed MTS choices made by memory and expect subjects at delays of $0,1,2$, and $4 \mathrm{sec}$ for the 6 test sessions, during which MTS trials were intermixed with probe test trials. As the delay between the removal of the compound sample and the opportunity to peck one of two comparison stimuli increased, the accuracy of MTS performance decreased in both subexperiments. Moreover, the accuracy of the expect subjects exceeded that of the memory subjects at all four delays.

Despite procedural differences between Experiments $1 \mathrm{~A}$ and $1 \mathrm{~B}$ with respect to the order in which the elements of the sample (a line orientation cue and a foodbased cue) were presented and the number of valid cues to the correct choice alternative, for purposes of statistical analysis, the memory and expect conditions, which defined the two subexperiments, were regarded as levels of a

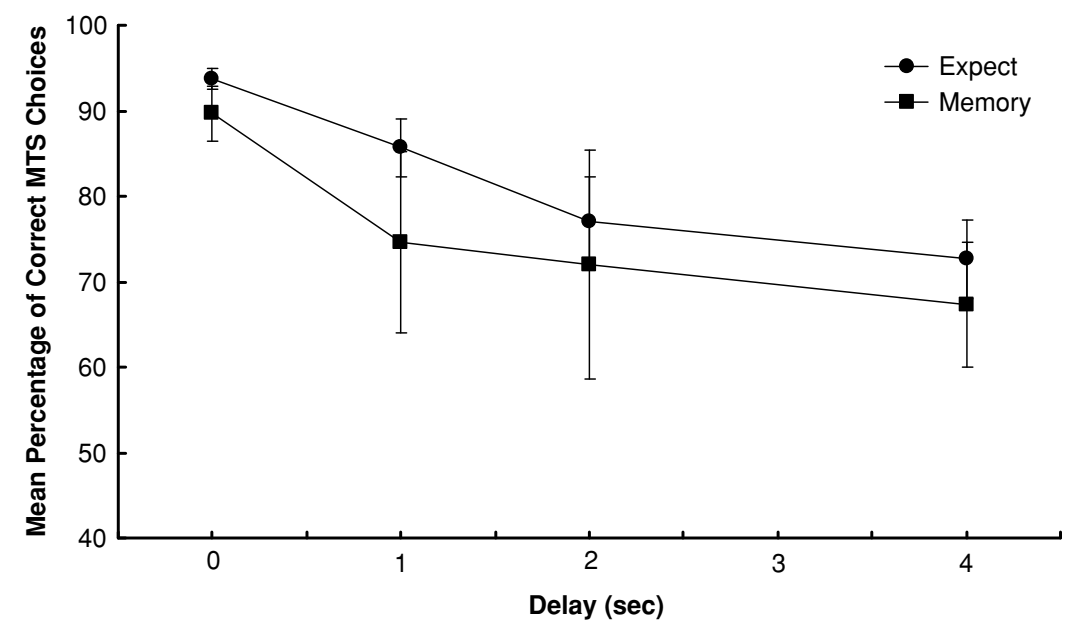

Figure 2. Experiment 1: Mean percentage of correct matching-to-sample (MTS) choices made by memory subjects and expect subjects on baseline trials at various delays. Plus and minus standard errors of the mean are indicated with error bars (unless the standard error is smaller than symbol size). 
single factor. A mixed two-way ANOVA was performed to evaluate the reliability of the effects displayed in Figure 2. The factors in the ANOVA included condition and delay. A .05 level of significance was employed for this analysis and for all subsequent analyses. The results of the ANOVA confirmed that conditional choice performance deteriorated with increasing delays between the removal of the sample and the choice opportunity $[F(3,12)=10.99, p=$ $.001]$. Neither the effect of condition nor the condition $\times$ delay interaction was significant, however $[F(1,4)$ and $F(3,12)<1$, respectively].

The mean rates of responding to the discriminative autoshaping stimuli (fields of different color) were approximately the same rate in both conditions.

\section{Test}

The transfer-of-control probe trials were identical in structure across the subexperiments and entailed the presentation of an autoshaping stimulus (i.e., the blue or the yellow field), followed by a choice between red and green. For purposes of calculating a measure of the control exerted by discriminative autoshaping stimuli on probe trials during testing, a pigeon's responses were considered "correct" when they were in the direction predicted by mediational theory. Specifically, for both Experiment 1A, which assessed transfer of control by substituting coloractivated representations of food for food-based memories, and Experiment 1B, which assessed transfer of control by substituting color-activated representations of food for food outcome expectancies, on probe trials beginning with blue, pecks directed at the green comparison stimulus were scored correct, whereas on probe trials beginning with yellow, pecks directed at the red comparison stimulus were scored correct. The probe trial performance of the memory and expect subjects is shown in Figure 3. As is evident from this figure, the autoshaping stimuli, which were correlated with the presentation of different grains, exerted immediate discriminative control over choices. It is noteworthy that these stimuli influenced the choice performance of the subjects assigned to Experiments 1A and $1 \mathrm{~B}$ in a similar manner, inasmuch as the choices by the pigeons in both subexperiments were consistent with predictions derived from mediational theory.

A mixed two-way ANOVA of the data shown in Figure 3 revealed no significant condition or delay effect and no condition $\times$ delay interaction $[F(1,4)=1.03, F(2,8)=$ 1.62 , and $F(2,8)=2.62$, respectively; all $p \mathrm{~s}>.10]$. To verify that reliable transfer of control occurred, the probe trial data, collapsed across subexperiments, were tested against chance $(50 \%)$ at each delay. The mean percentages of correct choices on probe trials at delays of 1,2 , and $4 \mathrm{sec}$ were $69.5 \%, 66.7 \%$, and $58.3 \%$, respectively. Onesample $t$ tests indicated reliable transfer at the 1- and 2-sec delays $[t(5)=5.53$ and $t(5)=2.74]$, but not at the $4-\mathrm{sec}$ delay $[t(5)=1.58]$. Given the variation in probe trial performance across delays within each condition, however, the extent of transfer in one condition, relative to the other, was not obvious.

\section{Response Mediation}

The remaining analyses relate to the possibility that the samples used in MTS training engendered different rates of pecking at the response key because of their relation to a unique food-based element of the sample in the memory condition or because of their relation to a particular food outcome event in the expect condition. If the autoshaping stimuli also elicited different rates of pecking by virtue of their association with food-related events that were common to the MTS task and the autoshaping procedure, the similarity in rates of responding to MTS samples and probe stimuli could provide a basis for guiding choices (Urcuioli, 1984; Urcuioli \& DeMarse, 1994).

As a first step in evaluating response mediation as an alternative to an explanation of transfer of control that is

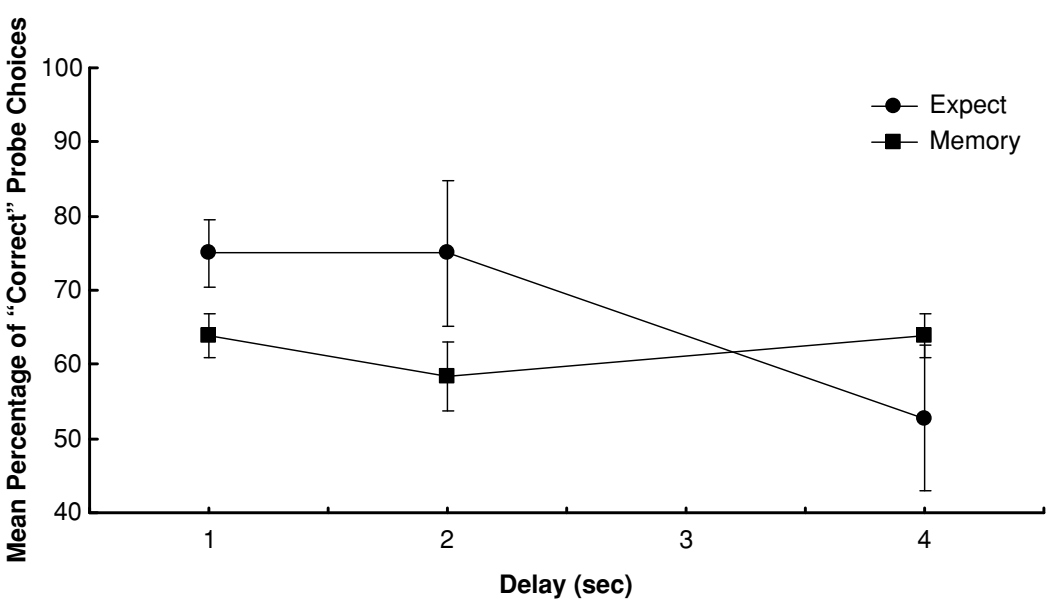

Figure 3. Experiment 1: Probe trial performance of memory and expect subjects. Responses were considered correct when they were in the direction predicted by mediational theory (see the text for a more detailed exposition of the scoring procedure). Plus and minus standard errors of the mean are indicated with error bars. 
based on cognitive mediation, the mean rates of responding to the autoshaping stimuli, which served as probes on probe trials, were calculated for each subject and condition. For the memory condition in Experiment 1A, the mean rates of pecking at the blue and the yellow fields were $1.17 \mathrm{pecks} / \mathrm{sec}(S E=0.51)$ and $0.93 \mathrm{pecks} / \mathrm{sec}$ $(S E=0.38)$, respectively. The corresponding means for the expect condition in Experiment 1B were 0.92 ( $S E=$ $0.29)$ and $0.91(S E=0.41)$. A mixed two-way ANOVA involving condition and stimulus as factors suggested no significant difference with respect to the rates at which the pigeons in either subexperiment pecked at the two autoshaping stimuli [for the condition and stimulus effects and the stimulus $\times$ condition interaction, $F(1,4)<1]$. Therefore, the results of our initial analysis did not corroborate a response mediation hypothesis.

Presumably, a higher rate of responding to one stimulus than to another reflects a preference for the grain with which this stimulus is correlated (e.g., in the case of a higher rate of pecking at blue than at yellow, a preference for safflower over milo). The analysis above assumes that all the pigeons had the same grain preference and, hence, responded more to stimuli associated with this grain. It is possible, however, that all the pigeons did not share a common preference. Although preferences and the resulting differences in rates of pecking may have varied from one pigeon to the next, they still could have served as the basis for transfer of control in individual pigeons - provided the latter consistently preferred one grain to another and, hence, pecked the stimulus predicting this grain at a higher rate. Thus, a second step in evaluating the response mediation hypothesis was to examine the probe trial performance of the individual subjects in Experiments 1A and 1B.

The purpose of our second analysis was to determine whether, in individual subjects and across test sessions, large differences in rates of responding to the two autoshaping stimuli were associated with more accurate probe trial performance and small differences in rates of responding were associated with less accurate probe trial performance. In essence, the question we posed was the following: Is there a correlation between the response rate differentials for the autoshaping stimuli and the conditional choice performance of a subject on probe trials? To address this question, the values of two variables in the 6 test sessions were ranked for each subject: (1) the magnitude of the difference between rates of responding to the autoshaping stimuli within a test session and (2) the percentage of correct choice responses on probe trials within a session. Spearman rank correlation coefficients over sessions were then calculated for the individual subjects in the memory and the expect conditions.

The correlation coefficients for the 3 subjects in the memory condition $(+.90,+.40$, and 0$)$ were generally, but not uniformly, positive and, thus, consistent with the notion that differential responding may have contributed to the transfer-of-control effect observed in Experiment 1A, at least to some degree. In contrast, the correlation coefficients for the 3 subjects in the expect condition $(+.24$,
+.02 , and -.38 ) ranged from positive to negative and were small in magnitude. When these coefficients are taken together, it seems unlikely that differential responding was responsible for the transfer-of-control effect observed in Experiment 1B. Across experiments, the data suggest that a response mediation account is lacking, for such an account potentially explains the probe test performance of only 2 subjects. An account of transfer based on mediation by cognitive processes - that is, associatively activated memories and food outcome expectancies - seems to provide a more consistent explanation of the data by predicting the transfer performance of all 6 subjects.

\section{EXPERIMENT 2}

Experiment 2 was designed to conceptually integrate Experiments 1A and 1B. In contrast to Experiment 1, which entailed comparisons between subexperiments, this experiment directly assessed the predominant nature of representations activated by stimuli. The primary question was the following: When a representation corresponding to a particular food-related event is activated associatively by a stimulus, is the representation more like a memory of that event or an expectancy of that event?

The subjects were trained on an MTS task involving only food and no-food events as samples. The food event was illumination of the hopper light concomitant with presentation of grain; the no-food event was illumination of the hopper light alone (as per Experiment 2 in Steirn et al., 1991). On food sample trials, correct responses to one color produced a no-food outcome; on no-food sample trials, responses to another color produced food. Thus, the food-based event that served as a sample was opposite the food outcome event that served as a reinforcer (see Dickinson \& de Wit, 2003, for a similar design).

After meeting the MTS criterion, the subjects received differential autoshaping. On autoshaping trials, two different stimuli were followed by food and no food. Therefore, events that served as samples and as outcomes in the MTS task were also used as reinforcers in differential autoshaping.

A small number of probe trials involving the autoshaping stimuli were introduced in the third phase of the experiment. The structure of these trials was similar to that of MTS trials. Probe trials were designed to determine whether associatively activated representations of food-related events more closely resembled (1) their selfprimed counterparts in the MTS task (i.e., memories of food and no-food samples) or (2) outcome expectancies (i.e., expectancies of food and no-food consequences). Figure 4 summarizes the design of Experiment 2.

Experiments 1A and 1B showed that associatively activated representations of food-related events are capable of functioning either like memories or like expectancies in guiding conditional choices. The design of Experiment 2 pitted these processes against each other. To make but one additional point regarding the design, Zentall et al. (1989) have reported that the discriminability of conditional cues 
Phase 1: Events Making Up Symbolic Delayed MTS Trials During Initial Training

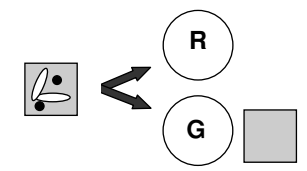

Sample Outcome

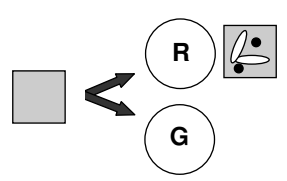

Sample Outcome

Phase 2: Differential Autoshaping Trials

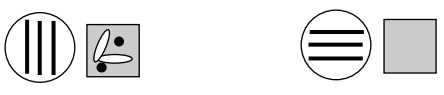

Phase 3: Transfer Testing

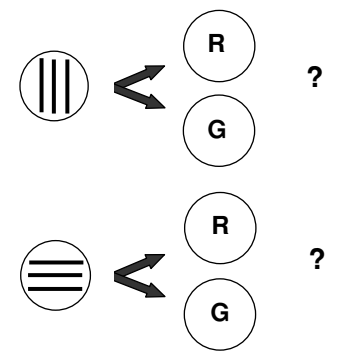

Figure 4. Experiment 2: Three phases of the transfer-of-control design. The features depicted in squares are mixed grain. $R$, red; $G$, green.

in a conditional choice task influences whether retrospective or prospective coding occurs - that is, whether a pigeon uses a representation of the sample or a representation of the correct comparison stimulus to bridge the retention interval in a symbolic delayed MTS task. Because the events used as samples and outcomes in our second experiment were identical - and perforce, equally discriminable - the design of this experiment obviated the influence of stimulus discriminability on type of coding.

\section{Method}

\section{Subjects}

The subjects were 3 female White Carneaux pigeons. They were obtained from Palmetto Pigeon Plant and were maintained under the same conditions as those described in the Method section for Experiment 1.

\section{Apparatus}

The three operant chambers used in Experiment 1 were also used in this experiment. Only the center grain feeder was employed.

\section{Procedure}

Preliminary training (autoshaping). The preliminary training phase of this experiment was similar to that in Experiment 1. The subjects received a maximum of 7 autoshaping sessions.

Symbolic delayed MTS training. Following autoshaping, the pigeons advanced to symbolic delayed MTS training. At the beginning of an MTS trial, a sample consisting of either a food or a nofood event was presented. The food event included the illumination of the aperture for $3 \mathrm{sec}$ concomitant with access to mixed grain. The no-food event also included illumination of the aperture for $3 \mathrm{sec}$, but the feeder did not operate, so grain was not available.

The sample was followed by the projection of red and green fields onto the left and right response keys. On the 24 trials beginning with the food sample, green was arbitrarily designated correct; a single peck to this stimulus extinguished both colors and resulted in the illumination of the aperture for $3 \mathrm{sec}$ without access to mixed grain - a consequence some would call secondary reinforcement. On the 24 trials beginning with the no-food sample, red was correct; thus, a peck to this stimulus resulted in aperture illumination and 3 -sec access to mixed grain - a consequence some would call primary reinforcement. A $15-\mathrm{sec}$ ITI ensued at the end of every trial.

Incorrect choices initiated a correction sequence similar to that described in Experiment 1. The subjects received 4 sessions of symbolic matching per week. These sessions were distributed throughout the week, and each session included 48 trials.

Symbolic delayed MTS training and differential autoshaping. Symbolic delayed MTS training with a delay of $0 \mathrm{sec}$ continued until a subject's average choice accuracy exceeded $90 \%$ for two consecutive 6-session blocks. Differential autoshaping trials were then interspersed among MTS trials, as in Experiment 1. Autoshaping trials began with the presentation of a set of vertical lines or horizontal lines on the center response key. The duration of the presentation of these line stimuli was $5 \mathrm{sec}$, and they were followed by food and no food, respectively - that is, by the same food-related events as those used in MTS training.

The subjects progressed from concurrent differential autoshaping and symbolic delayed MTS training with a delay of $0 \mathrm{sec}$ to concurrent differential autoshaping and symbolic delayed MTS training with a delay of $1 \mathrm{sec}$, then $2 \mathrm{sec}$, and then $4 \mathrm{sec}$, and finally with mixed delays. The criterion for advancing from one value of the delay between the sample and the comparison stimuli to the next (and ultimately, to 
mixed delays) was a choice accuracy of $90 \%$ for one 3 -session block. The conditions of MTS training with constant delays and with mixed delays were identical with one exception. During mixed-delay training, the delay between the sample and the comparison stimuli varied unpredictably over trials. Delays of $0,1,2$, and $4 \mathrm{sec}$ were used for the 9 sessions preceding the initial probe test.

Initial probe test. The probe test was designed to assess whether the representations activated by the differential autoshaping stimuli resembled the MTS food and no-food events in their capacity as food-based sample memories or resembled these MTS events in their capacity as food outcome expectancies. Except for the addition of a small number of probe trials and the use of refresher sessions to forestall the pigeons' eventual discrimination between training and test trials, the conditions of training during this phase of the experiment were identical to those described above.

Probe trials began with the presentation of a set of vertical or horizontal lines on the center response key for $5 \mathrm{sec}$. Two, 4 , or $8 \mathrm{sec}$ following the offset of this stimulus, a pigeon was confronted with a choice between red and green fields on the side keys. A response to one of these colors extinguished both of them and initiated the ITI. Extinction contingencies were in effect on these trials, as opposed to the MTS and differential autoshaping trials.

This phase of the experiment, which was similar to that described in Experiment 1, included a total of 12 probe test sessions. Every 2 test sessions were followed by a refresher session consisting of only differential autoshaping trials and symbolic delayed MTS trials with mixed delays.

Symbolic delayed MTS retraining, trace differential autoshaping, and probe test. After the initial probe test, the subjects received 12 sessions of differential autoshaping concurrent with MTS training. The conditions of training during this phase differed from the conditions preceding the initial probe test in that trace autoshaping trials with intervals greater than $0 \mathrm{sec}$ were interspersed among symbolic delayed MTS trials with mixed delays. The introduction of trace intervals greater than $0 \mathrm{sec}$ rendered the autoshaping trials more similar to the symbolic delay MTS and probe test trials. Autoshaping trials began with the presentation of a set of vertical lines or horizontal lines on the center response key for $5 \mathrm{sec}$. These vertical and horizontal stimuli were followed by food and no food, respectively, after a trace interval of $0,1,2,4$, or $8 \mathrm{sec}$. Each of the $0-, 1-$, and 2-sec intervals was used four times within a session (twice per line orientation stimulus). The 4 - and 8 -sec intervals were each used twice - that is, once with the horizontal stimulus and once with the vertical stimulus.

A second probe test was conducted subsequent to concurrent trace differential autoshaping and symbolic delayed MTS retraining with mixed delays. The second probe test resembled the first, except for the autoshaping procedure and the number of test sessions.

\section{Results and Discussion}

Our analyses focus on the question of whether the representations activated by the differential autoshaping stimuli on probe test trials more closely resembled memories of the food-based events that were used as samples in the MTS task or expectancies of the food outcome events that were used as reinforcers.

\section{Acquisition}

The subjects required a mean of 62.3 sessions $(S E=$ 4.26) to meet the criterion for symbolic delayed MTS training with a delay of $0 \mathrm{sec}$ - that is, choice accuracy of $90 \%$ across two consecutive 6 -session blocks. Subsequently, they required a mean of 4.7 sessions $(S E=1.67), 3.0$ sessions $(S E=0.00)$, and 5.7 sessions $(S E=1.77)$, respectively, to meet the MTS criterion of $90 \%$ accuracy for one 3-session block with constant delays of 1,2 , and $4 \mathrm{sec}$.

\section{Baseline}

Figure 5 displays the MTS performance of the subjects for the first and second series of test sessions, during which MTS trials were intermixed with probe test trials and differential autoshaping trials. In contrast to Exper-

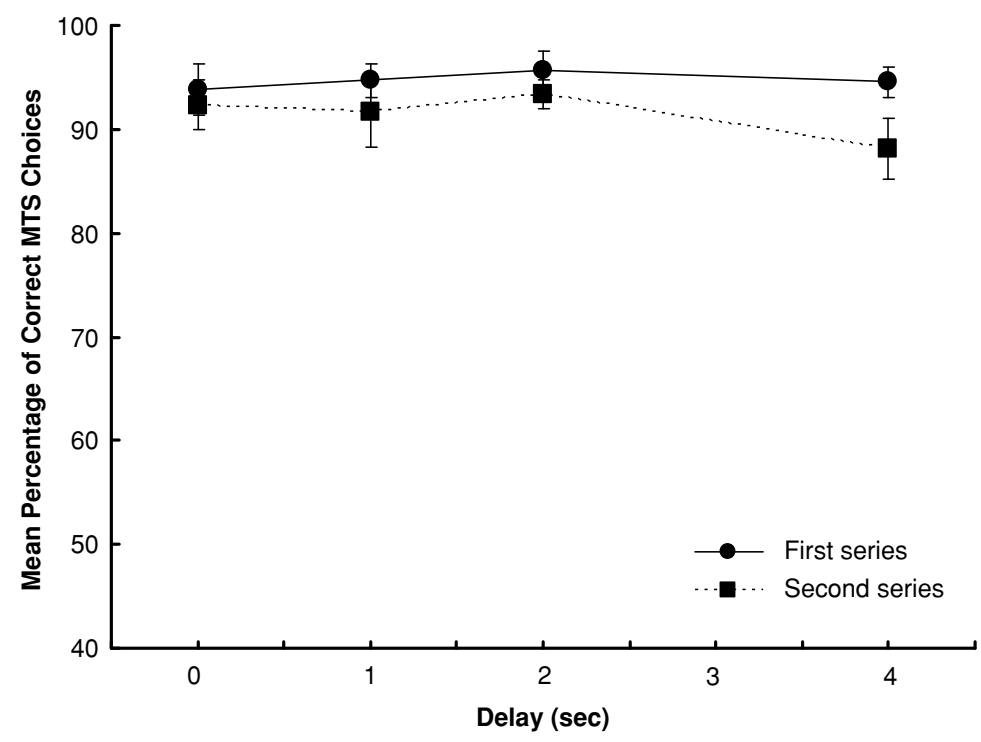

Figure 5. Experiment 2: Mean percentage of correct matching-to-sample (MTS) choices on baseline trials at delays of $0,1,2$, and $4 \mathrm{sec}$ for the first series of test sessions (solid line) and the second series of test sessions (dashed line). Plus and minus standard errors of the mean are indicated with error bars (unless the standard error is smaller than symbol size). 
iments $1 \mathrm{~A}$ and $1 \mathrm{~B}$, in which MTS performance deteriorated sharply with increasing delays between the removal of the sample and the choice opportunity, in the present experiment, the pigeons chose at relatively high levels of accuracy regardless of the retention interval in both tests. A two-way ANOVA involving test series and delay as factors confirmed the absence of any effects [test series, $F(1,2)=1.78$; delay, $F(3,6)=1.18$; test series $\times$ delay interaction, $F(3,6)=0.90$; all $p \mathrm{~s}>.30$ ].

\section{Test}

There were two obvious predictions regarding the pattern of choices by the pigeons during the test sessions, and both choice patterns lend themselves to interpretation in terms of mediational theory. If, on probe trials, the vertical stimulus (which activated a representation of food) directed responses to the green comparison and the horizontal stimulus (which activated a representation of no food) directed responses to the red comparison-just as, on MTS trials, the food and no-food samples directed responses to green and red, respectively - this pattern would indicate that associatively activated representations closely resemble sample memories. Alternatively, if the vertical stimulus directed responses to the red comparison, which was consistently followed by food on MTS trials, and the horizontal stimulus directed responses to the green comparison, which was consistently followed by no food, this pattern would indicate that associatively activated representations closely resemble food outcome expectancies.

For purposes of statistical analysis, on probe trials beginning with the vertical stimulus, choice of the red comparison was scored "correct," whereas on probe trials beginning with the horizontal stimulus, choice of the green comparison was scored "correct." Given this scoring system, mediation by food outcome expectancies is inferred when the proportion of correct choices is greater than chance, whereas mediation by food-based sample memories is inferred when the proportion of correct choices is less than chance.

The mean proportion of correct probe test choices at delays of 2, 4, and $8 \mathrm{sec}$ is shown in Table 2. Although the data for the first and second series of test sessions are shown separately at each delay, a two-way ANOVA indicated that the effects of test series and delay and the test series $\times$ delay interaction were not significant $[F(1,2)=$ $0.12, F(2,4)=1.10$, and $F(2,4)=3.22$, respectively; all $p \mathrm{~s}>$.15]. It is noteworthy, however, that all of the tabled proportions exceed chance. Therefore, the pattern of choices observed in Experiment 2 implies that associatively activated representations resemble food outcome expectancies and direct choices in a manner consistent with prospective mediation.

\section{Response Mediation}

In Experiments 1A and 1B, focused consideration was given to the possibility that the transfer-of-control effect observed was due to differential responding elicited by the samples and the autoshaping stimuli (i.e., to response mediation), rather than to cognitive processes. The designs of Experiments $1 \mathrm{~A}$ and $1 \mathrm{~B}$ were attempts to obviate the
Table 2

Mean Proportion of Correct Choices (Expectancy Based) During the Probe Test Sessions in Experiment 2

Test Series

\begin{tabular}{cccccc} 
& \multicolumn{3}{c}{ Test Series } \\
\cline { 2 - 5 } Delay (sec) & $M$ & $S E$ & & $M$ & $S E$ \\
\cline { 2 - 5 } \cline { 5 - 6 } & .64 & .08 & .70 & .07 \\
4 & .68 & .06 & .63 & .05 \\
8 & .67 & .06 & .56 & .06 \\
\hline
\end{tabular}

Note-"Correct" means that when autoshaping stimuli activated representations of food-related events, choices were consistent with mediation by food outcome expectancies (see the text).

contribution of differential responding to any transfer-ofcontrol effect by using equally preferred foods - safflower and milo - in both the MTS task and the autoshaping procedure. In the present experiment, however, food and no food were used in the MTS task and the autoshaping procedure. The subjects had an obvious preference for one of these food-related events and, in fact, responded at a higher rate to the vertical stimulus paired with food $(M=$ $2.10 \mathrm{pecks} / \mathrm{sec})$ than to the horizontal stimulus paired with no food $(M=0.02$ pecks $/ \mathrm{sec})$. Given this difference in rates of responding to the two stimuli, it is instructive to consider the pattern of probe test choices predicted by the response mediation hypothesis.

The pigeons pecked both the food sample and the vertical stimulus at a high rate, and they pecked both the nofood sample and the horizontal stimulus at a low rate. The food sample directed responses to the green comparison, and the no-food sample directed responses to the red comparison. Thus, if differential responding guided choices during the test sessions, on vertical probe trials, the pigeons should have pecked the green comparison, whereas on horizontal probe trials, they should have pecked the red comparison. This was not the pattern of probe test choices in Experiment 2; indeed, the pigeons tended to exhibit the opposite pattern. Therefore, the specific pattern of choices to probes indicated that associatively activated representations resembled prospective food outcome expectancies more closely than they resembled retrospective foodbased sample memories.

Before progressing to the general discussion, we should note that when the vertical stimulus and the horizontal stimulus were used to activate representations of foodrelated events on probe trials, the affective properties of these representations were likely more similar to those occasioned by food outcome expectancies than to those occasioned by food-based sample memories, and this may have influenced choices by the pigeons. Indeed, the relative contribution of affective and cognitive processes to transfer of control is an intriguing empirical issue, albeit beyond the scope of this study.

\section{GENERAL DISCUSSION}

Given the mediation of choices in delayed MTS tasks by retrospective memories and prospective expectancies 
of food-related events, our experiments addressed a seminal question: When Pavlovian stimuli are used to associatively activate representations of food-related events, are these representations more similar to food-based sample memories or to food outcome expectancies? The somewhat greater level of transfer observed in Experiment 1B, relative to $1 \mathrm{~A}$, suggests that the degree of resemblance between two associatively activated representations of a particular food-related event (e.g., a representation of food activated by an autoshaping stimulus and an outcome expectancy of food activated by a sample) is higher than the degree of resemblance between an associatively activated representation of a food-related event and a directly activated representation of this same event (e.g., a representation of food activated by an autoshaping stimulus and a representation activated by presentation of the food per se).

In addition to revealing the similarity between directly activated and associatively activated representations and between one associatively activated representation and another, the results of Experiments $1 \mathrm{~A}$ and $1 \mathrm{~B}$ corroborate findings reported by Urcuioli (1990) and by Steirn et al. (1991). As the reader will recall, Urcuioli (1990) demonstrated transfer of discriminative control over choices from samples to stimulus-activated representations of food-related events in an MTS task involving outcome expectancies as the functional cue, whereas Steirn et al. demonstrated transfer of discriminative control over choices from samples to stimulus-activated representations in an MTS task involving food-based memories. In contrast to the former studies, in which food-related events were distinguished by presenting grain with different probabilities, in Experiments 1A and 1B, two grains were used. This expedient was intended to equate the incentive value of the reinforcers common to the discriminative autoshaping procedure and the symbolic delayed MTS task and, thereby, to equate rates of responding to the stimuli associated with these reinforcers. The results of analyses undertaken to evaluate an alternative explanation for the transfer effects observed in the present study - namely, response mediation-confirmed that these effects were based on features that the stimulus-activated representations shared with memories of food-based samples (Experiment 1A) and with expectancies of food outcomes (Experiment 1B), as opposed to differential overt responding.

In the symbolic delayed MTS task in Experiment 2, each of the samples (food and no food) predicted a food outcome event with a different valence (no food and food, respectively). Differential autoshaping stimuli were also correlated with these outcomes, and when samples were replaced by autoshaping stimuli on probe test trials, control over choices transferred from the samples to the autoshaping stimuli. This transfer effect implies (1) that differential outcome expectancies cued choice responses during the symbolic delayed MTS training of Phase 1, (2) that the food-based events used as samples activated outcome expectancies of a very different kind, and (3) that representations of no food and food, which were activated asso- ciatively by autoshaping stimuli, resembled food outcome expectancies. In addition, Experiment 2 demonstrates that when one biologically relevant event is used to activate a representation of another (e.g., when food is used to activate a representation of no food), the activated representation may be indistinguishable from one evoked by a neutral event. Finally, the results of Experiment 2 indicate that under the present conditions in which retrospective and prospective coding are placed in opposition, animals may have a prospective coding bias.

We might note, parenthetically, that both sampleoutcome associations and comparison-outcome associations are established in a two-choice conditional discrimination involving food and no food as outcomes. Only the former, however, contribute to the superior performance of pigeons trained in a symbolic delayed MTS task involving different outcomes, due to the cuing function of sampleelicited expectancies (Sherburne \& Zentall, 1998).

The present experiments underscore the utility of conjoining delayed conditional discrimination training with transfer-of-control designs in the study of animal coding processes. Collectively, our results indicate that when a representation of some food-related event is activated associatively by a stimulus, the representation is more like an expectancy (prospective code) than it is like a memory (retrospective code) of this event. Experimental replication across a wider range of conditions and a variety of species would strengthen these conclusions. Moreover, it remains to be determined whether the prospective nature of associatively activated representations is unique to food-related events or is characteristic of events in general.

\section{REFERENCES}

Brodigan, D. L., \& Peterson, G. B. (1976). Two-choice conditional discrimination performance of pigeons as a function of reward expectancy, prechoice delay, and domesticity. Animal Learning \& Behavior, 4, 121-124.

Cook, R. G., Brown, M. F., \& Riley, D. A. (1985). Flexible memory processing by rats: Use of prospective and retrospective information in the radial maze. Journal of Experimental Psychology: Animal Behavior Processes, 11, 453-469.

Dickinson, A., \& DE WIT, S. (2003). The interaction between discriminative stimuli and outcomes during instrumental learning. Quarterly Journal of Experimental Psychology, 56B, 127-139.

GRANT, D. S. (1976). Effect of sample presentation time on long-delay matching in the pigeon. Learning \& Motivation, 1, 580-590.

Grant, D. S. (1993). Coding processes in pigeons. In T. R. Zentall (Ed.), Animal cognition: A tribute to Donald A. Riley (pp. 193-216). Hillsdale, NJ: Erlbaum.

Honig, W. K., \& DodD, P. W. D. (1986). Anticipation and intention in working memory. In D. F. Kendrick, M. E. Rilling, \& M. R. Denny (Eds.), Theories of animal memory (pp. 77-100). Hillsdale, NJ: Erlbaum.

Overmier, J. B., \& LaWry, J. A. (1979). Pavlovian conditioning and the mediation of behavior. In G. H. Bower (Ed.), The psychology of learning and motivation (Vol. 13, pp. 1-55). New York: Academic Press.

Peterson, G. B., Linwick, D., \& Overmier, J. B. (1987). On the comparative efficacy of memories and expectancies as cues for choice behavior in pigeons. Learning \& Motivation, 18, 1-20.

Rescorla, R. A., \& Solomon, R. L. (1967). Two-process learning theory: Relationships between Pavlovian conditioning and instrumental learning. Psychological Review, 74, 151-182.

Riley, D. A., CoOK, R. G., \& LAMB, M. R. (1981). A classification and 
analysis of short-term retention codes in pigeons. In G. H. Bower (Ed.), The psychology of learning and motivation (Vol. 15, pp. 5179). New York: Academic Press.

Roitblat, H. L. (1980). Codes and coding processes in pigeon shortterm memory. Animal Learning \& Behavior, 8, 341-351.

Roitblat, H. L. (1984). Representations in pigeon working memory. In H. L. Roitblat, T. G. Bever, \& H. S. Terrace (Eds.), Animal cognition (pp. 79-97). Hillsdale, NJ: Erlbaum.

RoITBLAT, H. L. (1993). Representations and processes in working memory. In T. R. Zentall (Ed.), Animal cognition: A tribute to Donald A. Riley (pp. 175-192). Hillsdale, NJ: Erlbaum.

Sherburne, L. M., \& Zentall, T. R. (1998). The differential outcomes effect in pigeons is not reduced by eliminating response-outcome associations: Support for a two-process account. Animal Learning \& Behavior, 26, 378-387.

Steirn, J. N., Jackson-Smith, P., \& Zentall, T. R. (1991). Mediational use of internal representations of food and no-food events by pigeons. Learning \& Motivation, 22, 353-365.

TRAPOLD, M. A. (1970). Are expectancies based upon different positive reinforcing events discriminably different? Learning \& Motivation, 1, 129-140.

Trapold, M. A., \& Overmier, J. B. (1972). The second learning process in instrumental learning. In A. H. Black \& W. F. Prokasy (Eds.), Classical conditioning II: Current research and theory (pp. 427-452). New York: Appleton-Century-Crofts.

UrCUIOLI, P. J. (1984). Overshadowing in matching-to-sample: Reduction in sample-stimulus control by differential sample behaviors. Animal Learning \& Behavior, 12, 256-264.

URCUIOLI, P. J. (1990). Some relationships between outcome expectancies and sample stimuli in pigeons' delayed matching. Animal Learning \& Behavior, 18, 302-314.

Urcuioli, P. J., \& DeMarse, T. (1994). On the relationship between differential outcomes and differential sample responding in matchingto-sample. Journal of Experimental Psychology: Animal Behavior Processes, 20, 249-263.
Urcuioli, P. J., Zentall, T. R., Jackson-Smith, P., \& Steirn, J. N. (1989). Evidence for common coding in many-to-one matching: Retention, intertrial interference, and transfer. Journal of Experimental Psychology: Animal Behavior Processes, 13, 264-273.

Wasserman, E. A. (1986). Prospection and retrospection as processes of animal short-term memory. In D. F. Kendrick, M. E. Rilling, \& M. R. Denny (Eds.), Theories of animal memory (pp. 53-75). Hillsdale, NJ: Erlbaum.

ZENTALL, T. R. (1998). Symbolic representation in animals: Emergent stimulus relations in conditional discrimination learning. Animal Learning \& Behavior, 26, 363-377.

Zentall, T. R., Hogan, D. E., \& Edwards, C. A. (1984). Cognitive factors in conditional learning in pigeons. In H. L. Roitblat, T. G. Bever, \& H. S. Terrace (Eds.), Animal cognition (pp. 389-405). Hillsdale, NJ: Erlbaum.

Zentall, T. R., Sherburne, L. M., \& Steirn, J. N. (1993). Common coding and stimulus class formation in pigeons. In T. R. Zentall (Ed.), Animal cognition: A tribute to Donald A. Riley (pp. 217-236). Hillsdale, NJ: Erlbaum.

Zentall, T. R., Steirn, J. N., \& Jackson-Smith, P. (1990). Memory strategies in pigeons' performance of a radial-arm-maze analog task. Journal of Experimental Psychology: Animal Behavior Processes, 16, 358-371.

Zentall, T. R., Urcuioli, P. J., Jackson-Smith, P., \& Steirn, J. N. (1991). Memory strategies in pigeons. In L. Dachowski \& C. F. Flaherty (Eds.), Current topics in animal learning: Brain, emotion, and cognition (pp. 119-139). Hillsdale, NJ: Erlbaum.

Zentall, T. R., Urcuioli, P. J., Jagielo, J. A., \& Jackson-Smith, P. (1989). Interaction of sample dimension and sample-comparison mapping on pigeons' performance of delayed conditional discriminations. Animal Learning \& Behavior, 17, 172-178.

(Manuscript received November 12, 2003; revision accepted for publication May 24, 2005.) 